\title{
Synaptic Convergence of Motor and Somatosensory Cortical Afferents onto GABAergic Interneurons in the Rat Striatum
}

\author{
Sankari Ramanathan, ${ }^{1}$ Jason J. Hanley, ${ }^{1}$ Jean-Michel Deniau, ${ }^{2}$ and J. Paul Bolam ${ }^{1}$ \\ ${ }^{1}$ Medical Research Council Anatomical Neuropharmacology Unit, Department of Pharmacology, Oxford, OX1 3TH, \\ United Kingdom, and 2Institut National de la Santé et de la Recherche Médicale U114, Collège de France, \\ 75321 Paris Cedex 05, France
}

\begin{abstract}
Cortical afferents to the basal ganglia, and in particular the corticostriatal projections, are critical in the expression of basal ganglia function in health and disease. The corticostriatal projections are topographically organized but also partially overlap and interdigitate. To determine whether projections from distinct cortical areas converge at the level of single interneurons in the striatum, double anterograde labeling from the primary motor (M1) and primary somatosensory (S1) cortices in the rat, was combined with immunolabeling for parvalbumin (PV), to identify one population of striatal GABAergic interneurons.

Cortical afferents from $\mathrm{M} 1$ and $\mathrm{S} 1$ gave rise to distinct, but partially overlapping, arbors of varicose axons in the striatum. PV-positive neurons were often apposed by cortical terminals and, in many instances, apposed by terminals from both cortical areas. Frequently, individual cortical axons formed multiple varicosities apposed to the same PV-positive neuron. Electron
\end{abstract}

The basal ganglia are a group of subcortical nuclei that are intimately involved in the control of movement. One of their major roles is to integrate sensory, motor, associative, and limbic information in the production of context-dependent behaviors (Graybiel, 1990, 1995). Anatomical and physiological data suggest that cortical information transmitted to the basal ganglia via the corticostriatal projection is channeled into parallel functional circuits that remain segregated at each level of the corticobasal ganglia-thalamo-cortical loops (Alexander et al., 1986, 1990; Alexander and Crutcher, 1990; DeLong, 1990; Hoover and Strick, 1993; Parent and Hazrati, 1995; Middleton and Strick, 2000). In addition to this organization underlying "parallel processing", the possibility for integration of diverse information within, and between, these loops also exists (Nauta and Domesick, 1984; Francois et al., 1987; Flaherty and Graybiel, 1991, 1993, 1995; Parthasarathy et al., 1992; Graybiel, 1995; Beiser et al., 1997; Maurin et al., 1999; Haber et al., 2000; Hoffer and Alloway, 2001; Kolomiets et al., 2001). Indeed, anatomical substrates that may underlie the integration of diverse information within the basal

Received May 20, 2002; revised June 21, 2002; accepted June 25, 2002.

This work was supported by the Medical Research Council (UK) and the European Community (BIOMED 2 Project: BMH4-CT-97-2215). We thank Caroline Francis and Paul Jays for technical support. We also thank Justin Boyes, Peter Magill, and Ahmed Sadek for their comments on this manuscript.

Correspondence should be addressed to J. P. Bolam, Medical Research Council Anatomical Neuropharmacology Unit, Department of Pharmacology, Mansfield Road, Oxford, OX13TH, UK. E-mail: paul.bolam@pharm.ox.ac.uk.

S. Ramanathan's present address: Department of Preclinical Veterinary Sciences, Royal (Dick) School of Veterinary Sciences, University of Edinburgh, Edinburgh, EH9 1QH, UK.

Copyright (ㄷ) 2002 Society for Neuroscience $\quad 0270-6474 / 02 / 228158-12 \$ 15.00 / 0$ microscopy confirmed that the cortical terminals formed asymmetric synapses with the dendrites and perikarya of PV-positive neurons as well as unlabelled dendritic spines. Correlated light and electron microscopy revealed that individual PV-positive neurons received synaptic input from axon terminals derived from both motor and somatosensory cortices.

These results demonstrate that, within areas of overlap of functionally distinct projections, there is synaptic convergence at the single cell level. Sensorimotor integration in the basal ganglia is thus likely to be mediated, at least in part, by striatal GABAergic interneurons. Furthermore, our findings suggest that the pattern of innervation of GABAergic interneurons by cortical afferents is different from the cortical innervation of spiny projection neurons.

Key words: GABA; striatum; corticostriatal; parvalbumin; cortex; basal ganglia

ganglia at the synaptic level have been identified (Somogyi et al., 1981b; Bevan et al., 1996, 1997). For instance, neurons of the substantia nigra pars compacta that project to the dorsal (motor and associative) striatum receive synaptic input from neurons located in the ventral (limbic) striatum (Somogyi et al., 1981b). Similarly, although there is a clear topography of the caudal projections of the ventral pallidum (limbic) and the globus pallidus (motor and associative), there are regions of overlap in the substantia nigra, subthalamic nucleus, and entopeduncular nucleus where synaptic convergence of the two divisions of the pallidal complex occurs at the single cell level (Bevan et al., 1996, 1997).

The striatum is also a site of functional convergence. Although the corticostriatal projections are highly topographically organized, they partially overlap and interdigitate (Malach and Graybiel, 1986; Gerfen, 1989; Flaherty and Graybiel, 1991, 1993, 1995; Parthasarathy et al., 1992; Brown et al., 1998; Takada et al., 1998; Hoffer and Alloway, 2001). Anatomical data suggests that corticostriatal projections from reciprocally connected cortical regions are more likely to have overlapping arborizations within the striatum (Yeterian and Van Hoesen, 1978; Pearson et al., 1983; Flaherty and Graybiel, 1993). Furthermore, projections from functionally related, but distinct, cortical regions (primary motor and primary somatosensory cortices) have been shown to converge in the striatum (Flaherty and Graybiel, 1993; Hoffer and Alloway, 2001). Electrophysiological analyses have shown striatal neurons to respond to both somatosensory and auditory stimuli in rats (Chudler et al., 1995) and to tactile, auditory, and visual stimuli in cats (Wilson et al., 1983b; Schneider, 1991). 


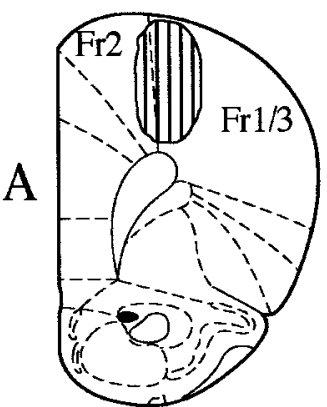

$3.20 \mathrm{~mm}$

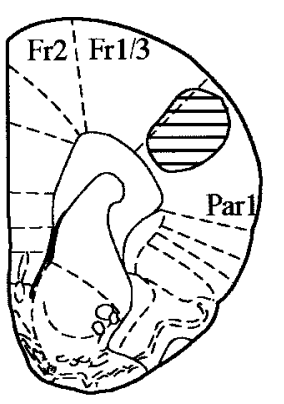

$2.20 \mathrm{~mm}$

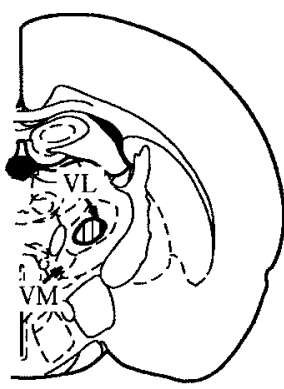

$-1.80 \mathrm{~mm}$

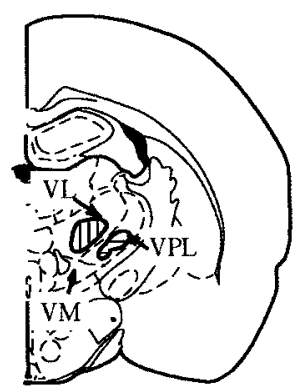

$-2.12 \mathrm{~mm}$

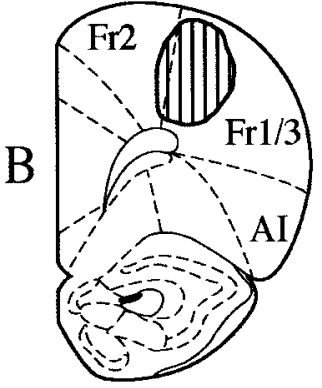

$3.70 \mathrm{~mm}$

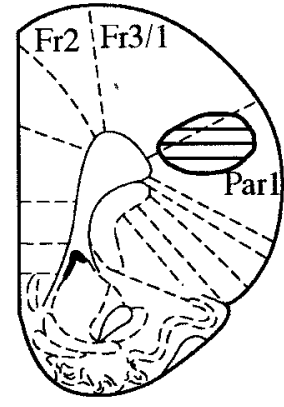

$2.70 \mathrm{~mm}$

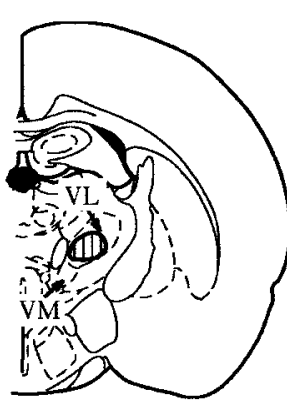

$-1.80 \mathrm{~mm}$

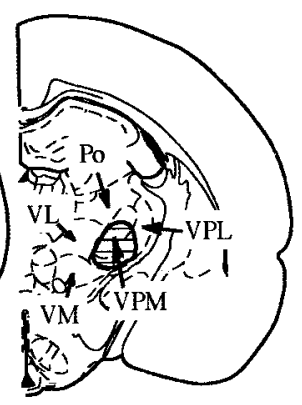

$-2.56 \mathrm{~mm}$

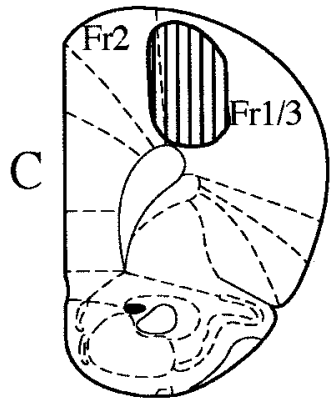

$3.20 \mathrm{~mm}$

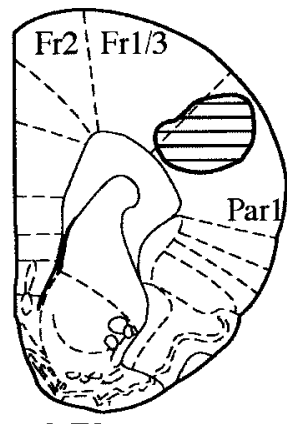

$2.70 \mathrm{~mm}$

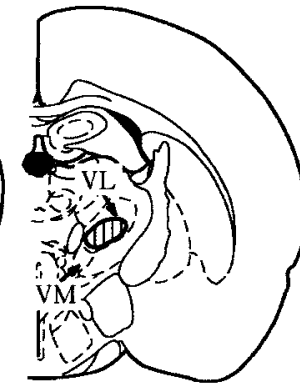

$-1.80 \mathrm{~mm}$

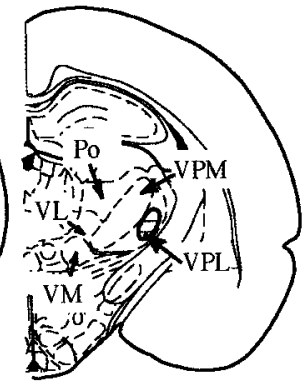

$-3.14 \mathrm{~mm}$
Figure 1. Schematic representations (modified from Paxinos and Watson, 1997) of the sites of injection of PHAL in M1 (vertical hatching) and BDA in S1 (horizontal hatching) and the corresponding labeling in the thalamus in the three animals used in the electron microscopic analysis. The deposits of PHAL were confined to frontal cortex areas 1 and 3 with a slight encroachment in area 2 . In each animal the thalamic labeling was confined to the ventrolateral nucleus of the thalamus. The BDA deposits were confined to parietal cortex, area 1, and labeling in the thalamus was confined to the lateral and medial aspects of the posterior nucleus. The figures denote the position in millimeters with respect to bregma (Paxinos and Watson, 1997). $V L$, Ventrolateral nucleus of thalamus; $V M$, ventromedial nucleus of thalamus; $V P L$, lateral aspect of ventroposterior nucleus of thalamus; $V P M$, medial aspect of ventroposterior nucleus of thalamus.
The main target of corticostriatal terminals are the spines of the GABAergic medium spiny projection neurons (Kemp and Powell, 1971b; Frotscher et al., 1981; Somogyi et al., 1981a; Dubé et al., 1988; Smith et al., 1994) and the cortical input shapes the activity of these neurons (Wilson et al., 1983a; Wilson, 1995; Wilson and Kawaguchi, 1996; Mahon et al., 2001). The second major target of the cortical input to the striatum is the class of GABAergic interneuron (Lapper et al., 1992; Bennett and Bolam, 1994) that expresses the calcium-binding protein parvalbumin (PV) (Cowan et al., 1990; Kita et al., 1990). The major target of these interneurons are the proximal regions of spiny projection neurons, and an individual interneuron may contact many hundreds of spiny neurons (Cowan et al., 1990; Kita et al., 1990; Kita, 1993; Bennett and Bolam, 1994). It has been proposed that they provide a feedforward inhibitory control of spiny neurons (Pennartz and Kitai, 1991; Jaeger et al., 1994; Kita, 1996; Plenz and Kitai, 1998) and indeed, they generate inhibitory synaptic potentials that are able to delay, curb, or possibly synchronize, the generation of action potentials in spiny projection neurons (Koos and Tepper, 1999).

In view of the critical position of PV-positive, GABAergic interneurons in the circuitry of the striatum and the fact that they are activated more easily and over a larger volume of striatum after cortical stimulation than are spiny neurons (Parthasarathy and Graybiel, 1997), we chose to analyze the cortical input to these neurons. Thus, the aims were to determine whether cortical afferents from the primary motor cortex (M1) and primary somatosensory cortex (S1) converge on individual PV-positive interneurons and to provide insight into the pattern of innervation of these neurons by individual cortical axons.

\section{MATERIALS AND METHODS}

Surgery. The experiments were performed on adult female Wistar rats (200-350 gm; Charles River, Margate, Kent, UK). Environmental conditions for housing of the rats and all procedures that were performed on them were in accordance with the Animals (Scientific Procedures) Act of 1986 and the policy on the use of animals issued by the Society for 


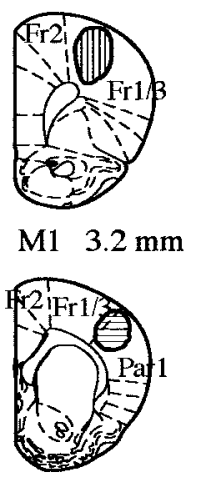

S1 $1.7 \mathrm{~mm}$

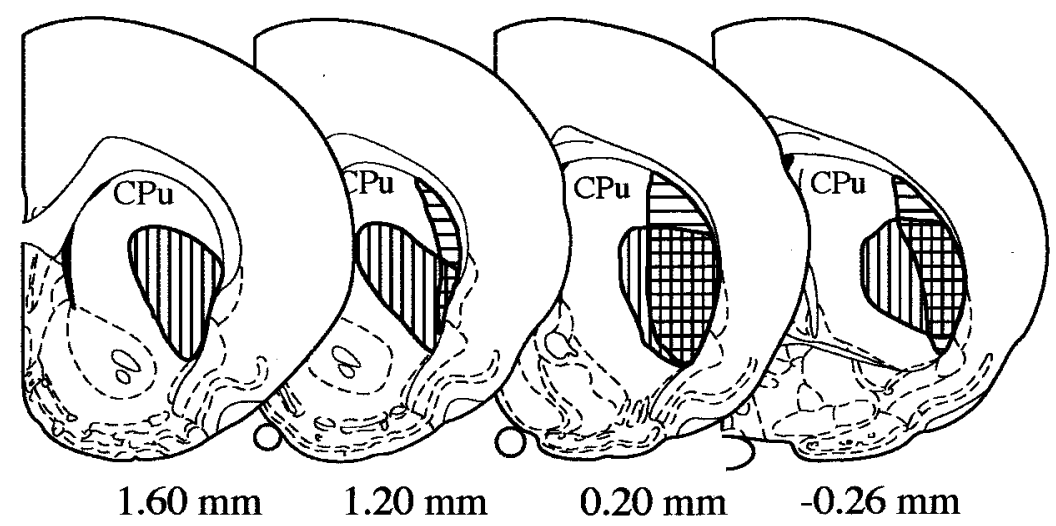

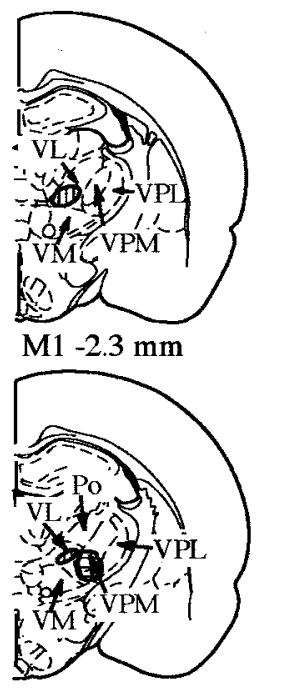

$\mathrm{S} 1-2.8 \mathrm{~mm}$
Figure 2. Schematic representations (modified from Paxinos and Watson, 1997) of the sites of injection of PHAL in primary motor cortex $(M 1)$ and BDA in the primary somatosensory cortex $(S 1)$, the corresponding labeling in the thalamus and the anterograde labeling in the striatum (caudateputamen; $C P u$ ). In each diagram the PHAL injection and the corresponding transport sites are indicated by vertical hatching, and the BDA injection and transport sites are indicated by horizontal hatching. The figures denote the position in millimeters with respect to bregma (Paxinos and Watson, 1997).

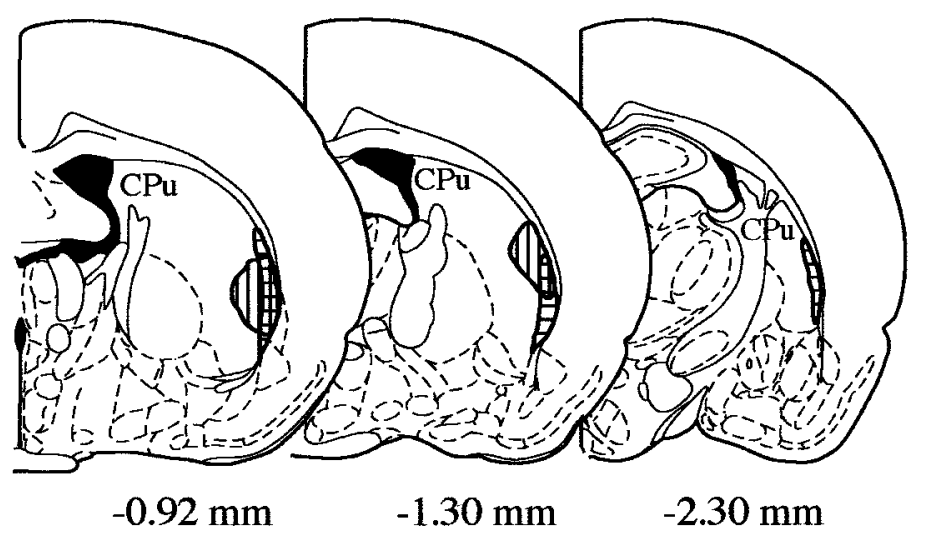

temperature. The injected and transported BDA was revealed using the avidin-biotin-peroxidase complex method (ABC; 1:100 in PBST-BSA; Vector Laboratories) with 3,3'-diaminobenzidine (DAB; $25 \mathrm{mg} / 100 \mathrm{ml}$ Tris buffer; Sigma, Dorset, UK; $0.006 \% \mathrm{H}_{2} \mathrm{O}_{2}$ ) as the chromogen for the peroxidase reaction. To reveal the injected and transported PHAL, sections were incubated overnight in rabbit anti-PHAL (1:1000 in PBSTBSA; Vector Laboratories), treated with goat anti-rabbit IgG (1:200 in PBST-BSA; Dako, High Wycombe, UK) for $2 \mathrm{hr}$, followed by a $1 \mathrm{hr}$ incubation in rabbit peroxidase-antiperoxidase (PAP) (1:100 in PBSTBSA; Dako), all at room temperature. The bound peroxidase was then revealed with $\mathrm{DAB}$ in the presence of nickel ions (nDAB). In some animals the BDA was revealed with $\mathrm{nDAB}$ and the PHAL with $\mathrm{DAB}$. Parvalbumin-immunoreactive structures were revealed by incubation in mouse anti-PV (1:1000 in PBST-BSA; Swant, Bellinzona, Switzerland) for $24-36 \mathrm{hr}$ at $4^{\circ} \mathrm{C}$ followed by goat anti-mouse IgG (1:200 in PBSTBSA; Jackson ImmunoResearch, West Grove, PA) for $2 \mathrm{hr}$ at room temperature and mouse PAP (1:100; Dako) with Vector SG as chromogen for the peroxidase reaction.

Preparation of tissue for electron microscopy. The striata of six animals were processed for electron microscopy. To increase the penetration of reagents, the sections were freeze-thawed in isopentane (BDH Chemicals, Poole, UK) cooled in liquid nitrogen up to three times. The sections were washed several times in PBS before the tracers and parvalbuminimmunoreactive structures were revealed. The method was as described

Figure 3. Light microscopy of convergence of motor and somatosensory afferents in the striatum: parvalbumin-immunostained perikarya $(P V)$ and axons anterogradely labeled with PHAL from M1 and axons anterogradely labeled with BDA from S1. In these cases the PHAL-containing motor cortical fibers were revealed using $\mathrm{DAB}$ as the chromogen for the peroxidase reaction giving a brown reaction product. The BDA-containing axons were labeled with nickel DAB as the chromogen giving the blue reaction product. These digital images and those in Figures $5, A$ and $D$, and $6, A$ and $D$, were prepared from scanned images of color photomicrographs and have been color balanced in Adobe Photoshop 6.0. They are derived from sections that were prepared for light microscopic analysis only. A, This parvalbumin-immunolabeled neuron (PV)is in a region containing (Figure legend continues.) 

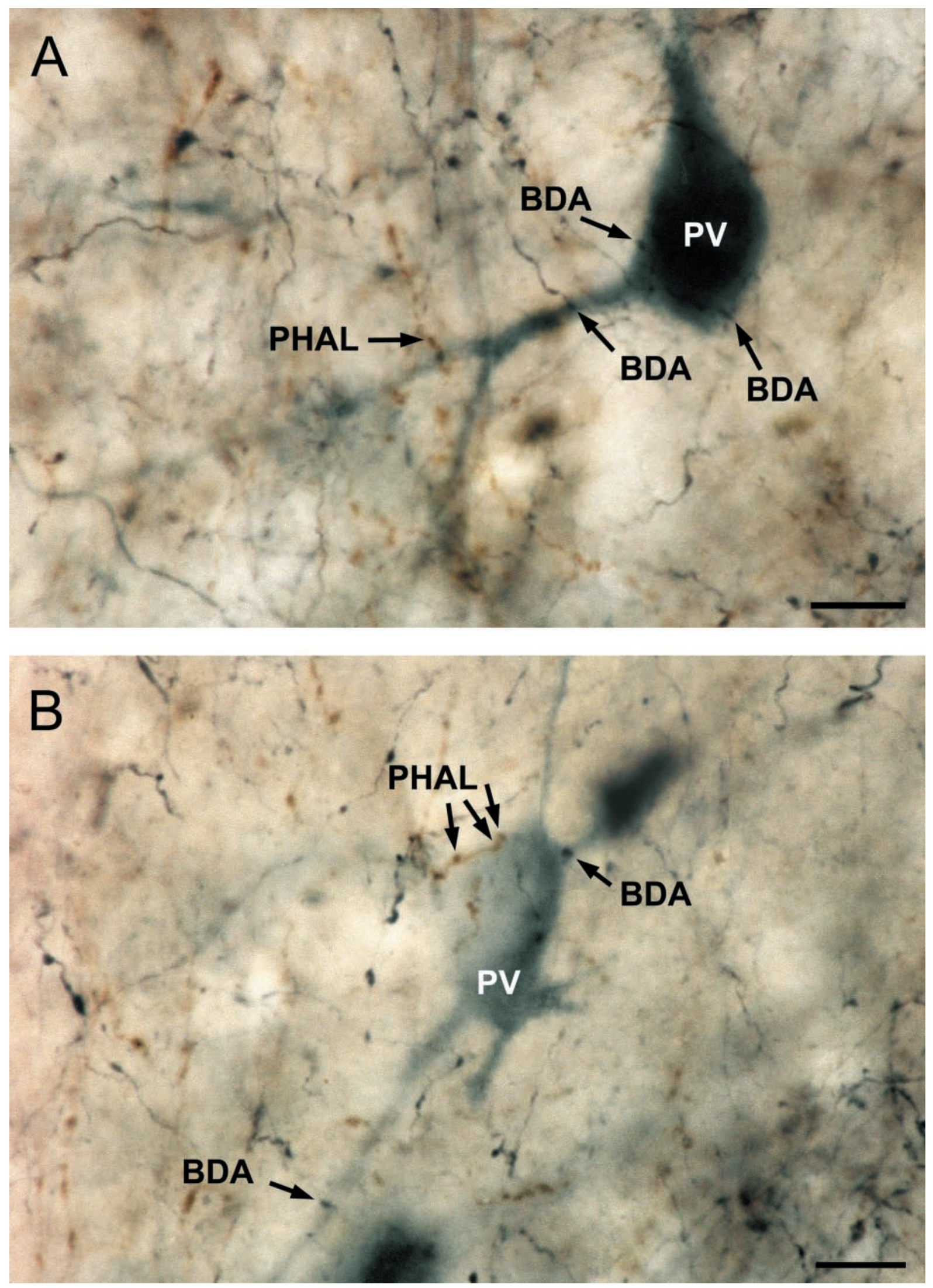

(Figure legend continued.) many PHAL- and BDA-labeled axons, many of which are closely apposed to the labeled neuron. At this focal depth there are several BDA-labeled boutons apposed to the perikaryon and proximal dendrite, and a PHAL-labeled bouton also closely apposes the dendrite. $B$, Montage of a second parvalbumin-immunolabeled neuron $(P V)$ that is apposed by boutons derived from the motor cortex (PHAL) and somatosensory cortex $(B D A)$. Note that the PHAL-labeled axon gives rise to several boutons that are apposed to the PV-positive perikaryon. Scale bars, $10 \mu \mathrm{m}$. 

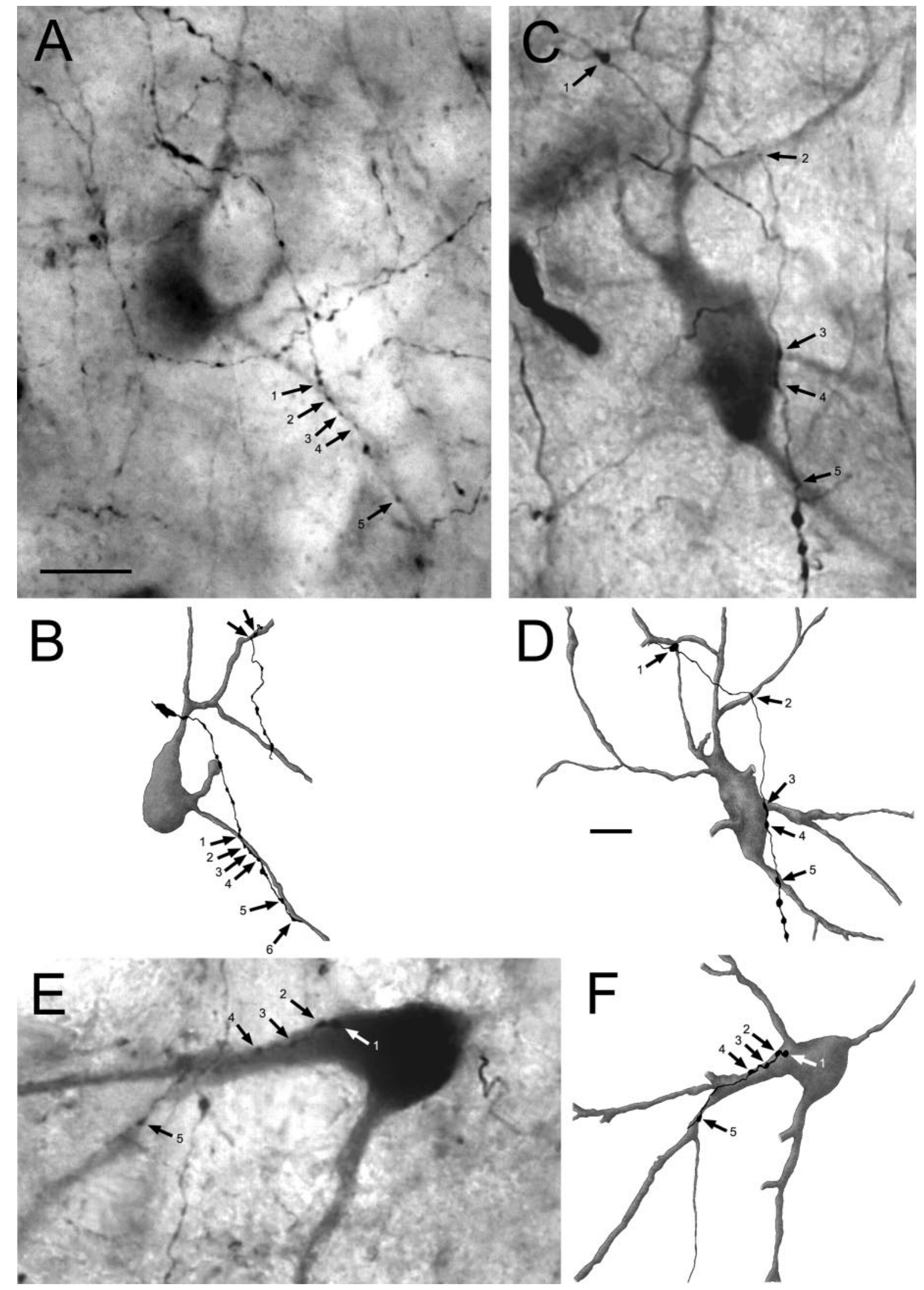

Figure 4. Light microscopic digital images and corresponding camera lucida drawings of striatal parvalbumin-positive neurons and individual cortical axons forming multiple appositions. $A, B$, Digital montage $(A)$ and drawing $(B)$ of a parvalbumin-positive interneuron and anterogradely labeled cortical fibers in the striatum. The PV-positive neuron gave rise to several immunolabeled dendrites, one of which was closely apposed by an axon anterogradely labeled with BDA from the somatosensory cortex. The axon gave rise to six varicosities that closely apposed the dendrite (Figure legend continues.) 
above with the omission of Triton X-100 from all solutions. The labeled sections of the striatum were postfixed in $1 \%$ osmium tetroxide (Oxkem), $5 \% \beta$-D-glucose (BDH Chemicals) in $0.1 \mathrm{M} \mathrm{PB}$ at $\mathrm{pH} 7.4$ for $60-70 \mathrm{~min}$. (Acsady et al., 1996). The sections were dehydrated through a graded series of dilutions of acetone (with 1\% uranyl acetate in the $70 \%$ solution) and infiltrated with resin overnight (Durcupan; Fluka Chemicals). They were then mounted in resin on glass microscope slides and polymerized at $60^{\circ} \mathrm{C}$ for $48 \mathrm{hr}$.

Analysis of material. All sections containing the sites of injection of the tracers were examined to ensure that they were correctly placed. The locations of the injection sites were also confirmed by analysis of sections of the thalamus for anterogradely and retrogradely labeled structures. Sections of the striatum from those animals in which the injections were correctly located were examined in the light microscope for the anterograde tracers and PV immunoreactivity. In some animals the anterograde labeling was plotted and recorded schematically. Particular attention was paid to regions of overlap of the two tracers. In these areas, PV-immunoreactive neuronal perikarya and emerging dendrites were examined at high magnification, and the positions of anterogradely labeled terminals closely apposed to them was noted. In some cases PV-positive neurons and individual cortical axons were drawn with the aid of a drawing tube and photographed digitally.

In a semiquantitative analysis, a single section of the striatum from three rats that were prepared for light microscopy (i.e., Triton X-100 included in the incubations) were analyzed at high magnification. The selected sections were those in which the region of overlap of the anterograde labeling from the two regions of the cortex was the most extensive. The location of each PV-positive perikaryon and emerging dendrites was noted. The proportion apposed by anterogradely labeled terminals derived from either region of the cortex was noted.

From the tissue that was processed for electron microscopy, eight PV-immunoreactive neurons (from four animals) whose cell bodies and/or dendrites were identified as being apposed by anterogradely labeled terminals from both regions of the cortex in the light microscope, were selected for further study. The cells were drawn and photographed at high magnification and examined by correlated light and electron microscopy. The coverslip overlying the tissue was removed using a razor blade. The area of interest was cut from the microscope slide and glued to the top of a blank cylinder of resin using cyanoacrylate glue. Serial ultrathin sections of 40-60 nm thickness were cut on a Reichert-Jung Ultracut E ultramicrotome (Leica, Nussloch, Germany) and collected on Pioloform-coated single slot copper grids. The ultrathin sections were then contrasted with lead citrate for 2-3 min and examined in a Philips CM 10 electron microscope.

\section{RESULTS}

\section{Light microscopic observations}

\section{Appearance of the reaction products}

The anterogradely labeled and immunolabeled structures were visualized with different chromogens for the peroxidase reactions that were distinguishable at the light microscopic level. Structures visualized with DAB as the chromogen for the peroxidase reaction were characterized by the presence of the typical reddish brown amorphous reaction product (see Fig. 3), and those visualized with nDAB contained the typical blue-black reaction product (see Fig. 3). Parvalbumin-immunoreactive structures were visualized using Vector SG as the chromogen and were characterized by the presence of a grayish blue reaction product that was less homogeneous that the DAB reaction products (see Fig. 3). The use of osmium tetroxide solution supplemented with glucose maintained color separation, at the light microscopic level, between different reaction products in the sections that were prepared for examination by both light and electron microscopy (Acsady et al., 1996) (see Figs. 5D, 6A,D).

\section{Injection sites}

The location of the injection sites of the two anterograde tracers (PHAL and BDA) was confirmed by visualization of the tracers in the M1 and S1 cortices (Figs. 1, 2). In the majority of cases the deposits of the two tracers were clearly separated. They spanned most of the cortical laminas without inclusion of the underlying corpus callosum. In some cases there were retrogradely labeled neurons of S1 close to the M1 injection site. However, these retrogradely labeled neurons were clearly separate from the filled neurons at the injection site; they constituted only very few neurons and were thus unlikely to influence the findings.

The location of the injections was confirmed by the analysis of the coincidental anterograde and retrograde labeling of thalamic nuclei. The motor cortex is innervated by thalamocortical projections mainly from the ventromedial and ventrolateral thalamic nuclei and in turn sends projections back to these motor nuclei (Cicirata et al., 1986, 1990). The somatosensory cortex is reciprocally connected to the ventrobasal nuclei, the intralaminal nucleus centralis lateralis, and the medial portion of the posterior thalamic group (Price and Webster, 1972; Nothias et al., 1988; Bourassa and Deschenes, 1995). In four of the six animals prepared for electron microscopy the labeling of thalamic nuclei was distinct for both the injection sites, and cells from these animals were studied at the ultrastructural level. In the remaining two animals there was clearly an overlap of the two injection sites in the different cortical territories as indicated by the thalamic labeling, these animals, and those prepared for light microscopy in which overlap of injections occurred, were excluded from the analysis.

\section{Distribution of anterograde labeling}

The deposits of PHAL and BDA in the M1 and S1 cortices, respectively, led to intense labeling of corticostriatal projections that were topographically organized and largely consistent with previous observations. The corticostriatal axons were collected in the fascicles of axon bundles traversing the striatum, and axonal arbors were primarily located around the fiber fascicles. The typical pattern of innervation of the striatum from M1 is illustrated in Figure 2. Anterogradely labeled fibers occurred in a band of striatum extending from $\sim 1.6 \mathrm{~mm}$ rostral of bregma to $\sim 1.3 \mathrm{~mm}$ caudal of it. Anterograde labeling from S1 occurred in a band in the lateral aspects of the striatum extending from $\sim 1.2$ $\mathrm{mm}$ rostral of bregma to $\sim 2.3 \mathrm{~mm}$ caudal. The band extended over a large part of the striatum in the dorsoventral plane and, at its maximum extent, occupied approximately one-third of the striatum in the mediolateral plane (Fig. 2).

A large part of the more lateral and caudal aspects of the projection from M1 overlapped with the projection from S1. In these regions the two sets of anterogradely labeled terminals were intermixed to such an extent that axonal varicosities derived from

\footnotetext{
(Figure legend continued.) (small arrows, 1-6), five of which are visible in the light micrograph (1-5). The neuron was also apposed by two varicosities of an axon anterogradely labeled from the motor cortex that is not visible at the focal depth of the micrograph but is shown in the drawing (two arrows, top right). $C, D$, A parvalbumin-positive neuron apposed by an axon anterogradely labeled with PHAL from the motor cortex. The axon give rise to five boutons that closely appose the dendrites and perikaryon of the labeled neuron. $E, F$, A parvalbumin-positive neuron apposed by an axon anterogradely labeled with BDA from the somatosensory cortex. The axon gave rise to five boutons that closely apposed the perikaryon and dendrites of the labeled neuron. Scale bars: $A, C, E, 10 \mu \mathrm{m} ; B, D, F, 10 \mu \mathrm{m}$.
} 

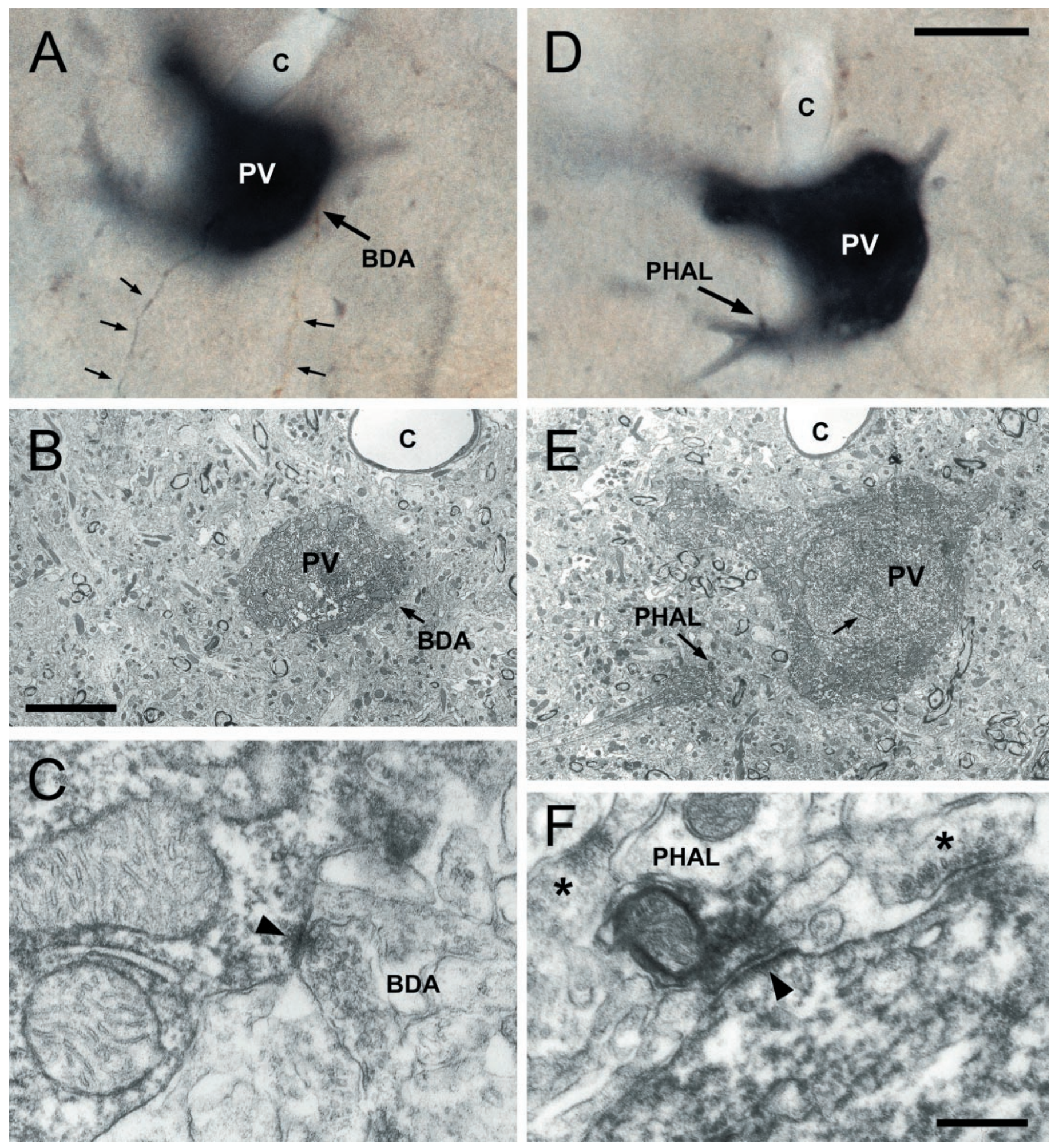

Figure 5. Synaptic convergence of motor and somatosensory cortical afferents onto a parvalbumin-positive, GABAergic interneuron in the striatum: correlated light and electron microscopy. $A$, Light micrograph of a parvalbumin-immunostained neuron $(P V)$ labeled using Vector SG as the substrate for the peroxidase reaction. The neuron was located in a region containing fibers anterogradely labeled with PHAL from the motor cortex (PHAL, nDAB as chromogen; blue fiber indicated by small arrows on the left) and with BDA from the somatosensory cortex (BDA, DAB as chromogen; brown fiber indicated by small arrows on the right). The axon from the somatosensory cortex gives rise to several varicosities, one of which closely apposes the perikaryon $(B D A$, large arrow). The capillary $(c)$ is labeled as a landmark between the light and electron microscopic levels. $B$, Low-power electron micrograph of part of the same perikaryon and the BDA-labeled, somatosensory cortical bouton closely apposed to it (BDA, arrow). $C$, High-power electron micrograph of the BDA-labeled bouton from the somatosensory cortex. The labeled terminal forms an asymmetrical synaptic contact (arrowhead) with the parvalbumin-immunolabeled neuron. $D$, Digital light micrograph of the same parvalbumin-immunolabeled neuron $(P V)$ at a deeper focal depth. At this level the neuron is apposed by a PHAL-positive bouton (PHAL, arrow) derived from the motor cortex. E, Low-power electron micrograph at about the same level as in D. The perikaryon, part of a dendrite, and the PHAL-labeled bouton (Figure legend continues.) 
the different cortical territories were often observed in close proximity (Figs. 3, 4B, 5A,D, 6A,D).

\section{Parvalbumin-positive GABAergic interneurons}

Parvalbumin-positive interneurons were identified by the gray reaction product formed by the Vector SG. Perikarya and proximal dendrites, as well as isolated dendrites, were labeled. Their morphology and distribution were consistent with previous studies (Cowan et al., 1990; Kita et al., 1990; Bennett and Bolam, 1994). The labeled neurons had medium-sized cell bodies, which were oval, or fusiform in shape. In some cases, indentations of the nuclear membrane were visible. Labeled primary dendrites branched close to the cell body and the secondary dendrites were generally smooth but sometimes gave rise to varicosities (Figs. 3, 4). Higher order dendrites were usually not labeled. The heaviest labeling of PV-positive structures was in the dorsolateral aspect of the striatum. Although the striatum is known to possess a dense network of PV-positive local axons and axonal boutons, PVpositive axonal fields were usually not labeled in the present study. This may reflect the sensitivity of the chromogen used and the fact that the immunostaining for PV was performed last.

Light microscopic analysis of convergence of cortical terminals on PV-positive neurons

Parvalbumin-positive neuronal perikarya and dendrites were intermingled among axons and axonal boutons anterogradely labeled from both M1 and S1 cortices (Fig. 3). The PV-positive structures were often closely apposed by the anterogradely labeled boutons, consistent with previous observations of cortical input to this class of neuron (Lapper et al., 1992; Bennett and Bolam, 1994). In many cases, an individual PV-positive neuronal perikaryon or isolated dendrite was closely apposed by terminals anterogradely labeled from M1 and terminals anterogradely labeled from S1 (Fig. 3). Examination of all PV-positive perikarya and emerging dendrites (but not isolated dendrites) in single sections at the level of the greatest extent of overlap of the two projections, revealed that up to $51 \%$ (range, 35.4-50.9) of PVpositive neurons were apposed by terminals derived from the cortex. Up to $46 \%$ (range, 24.7-46.2) of those that were apposed by cortical terminals were apposed by terminals derived from both cortical regions, which represents up to $23 \%$ (range, 8.823.5) of all PV-positive neurons in the single sections. In addition, single axons anterogradely labeled from either M1 or S1 were frequently found to form multiple appositions with individual PV-positive interneurons within a few microns (Fig. 4). They commonly gave rise to two or three boutons apposed to an individual PV-positive neuron, although as many as six were observed (Fig. 4).

\section{Electron microscopic observations}

To confirm that the appositions observed in the light microscopic analysis were indeed synapses, PV-positive neurons were examined by electron microscopy. Correlated light and electron microscopy was performed because the extent and quality of immunohistochemical and histochemical staining is reduced in material prepared for electron microscopy. A total of eight PV- positive neurons (two from each of the four rats) that were apposed by terminals from both M1 and S1 were selected at the light microscopic level for study in the electron microscope.

In the electron microscope the cell bodies and dendrites of the labeled PV-positive structures contained an amorphous, electrondense reaction product similar to that previously reported for Vector SG (Hussain et al., 1996; Hanley and Bolam, 1997). Ultrastructural features of the PV-immunoreactive structures were consistent with previous descriptions (Kita et al., 1990; Lapper et al., 1992; Bennett and Bolam, 1994). They possessed a relatively large volume of cytoplasm that was rich in organelles such as mitochondria, ribosomes, and Golgi apparatus (Figs. $5 B, C, E, F, 6 B, C, E, F)$. The nuclear membrane possessed indentations (Figs. $5 E, 6 E$ ), and intranuclear inclusions were often observed (Fig. 5E). Anterogradely labeled axon terminals were identified by the presence of reaction product as well as by their position in relation to landmarks such as blood vessels, unstained neurons, and glial cells (Figs. 5B,C,E,F, 6B,C,E,F). Axons and terminals that were visualized using $\mathrm{nDAB}$ were more intensely stained than DAB-labeled structures (compare Figs. 5C,F, 6C,F). Consistent with previous studies (Kemp and Powell, 1971a; Somogyi et al., 1981a; Dubé et al., 1988; Smith et al., 1994; Hersch et al., 1995) the anterogradely labeled corticostriatal boutons were packed with round vesicles and usually contained one or more mitochondria (Figs. 5C,F, 6C,F). They formed asymmetric synapses with dendritic spines and with dendritic shafts. The terminals were variable in size, and some of the larger boutons were similar in morphology to the boutons of the "discrete" corticostriatal projection from the barrel cortex (Wright et al., 1999). The correlated light and electron microscopy revealed that they also formed asymmetric synapses on the cell body and proximal dendrites of PV-positive interneurons (Figs. 5, 6). Of the eight cells studied, five of them (from three rats) were found to receive convergent synaptic input from both the M1 and S1 cortices (Figs. 5, 6). In one of the neurons that received the convergent input, three synapses from the motor cortex arose from a single axon.

Of the three neurons that were examined by correlated light and electron microscopy and failed to reveal convergent input from the cortex, two were abandoned because of poor ultrastructural preservation. In only one case was a labeled bouton identified at the light microscopic level found not to make contact with the PV-positive neuron; the bouton, anterogradely labeled from $\mathrm{S} 1$, made synaptic contact with an adjacent, unstained dendritic spine (data not shown).

In addition to the labeled boutons identified at both the light and electron microscopic levels, additional labeled boutons were observed in contact with the PV-positive neurons. These boutons $(n=6)$ had the morphology of corticostriatal boutons and formed asymmetric synapses, and they were usually found below the PVlabeled cell bodies. In addition to these, many unlabelled boutons formed asymmetric synaptic contacts with PV-immunolabeled cell bodies and dendrites.

\section{$\longleftarrow$}

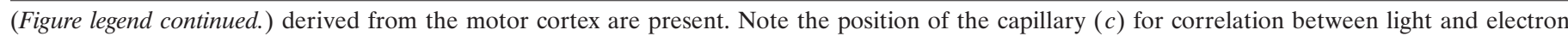

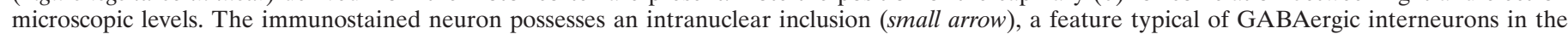

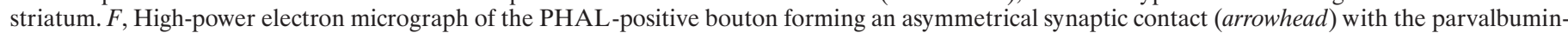

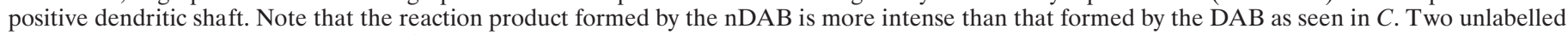
axonal boutons are indicated by asterisks. Scale bars: $A, D, 12.5 \mu \mathrm{m} ; B, E, 5 \mu \mathrm{m} ; C, F, 0.25 \mu \mathrm{m}$. 

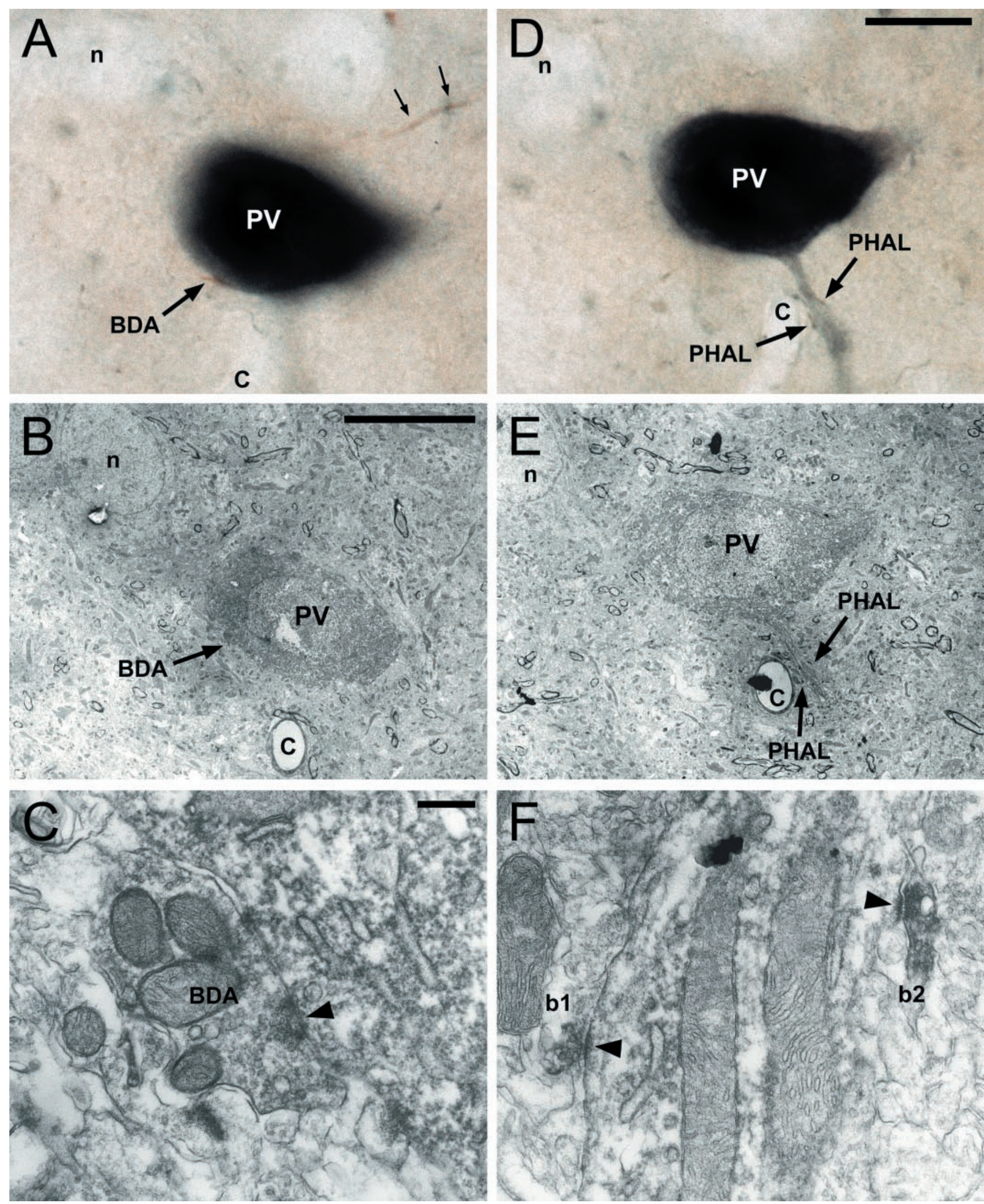

Figure 6. Synaptic convergence of motor and somatosensory cortical afferents onto a parvalbumin-immunolabeled, GABAergic interneuron in the striatum: correlated light and electron microscopy. $A$, Light micrograph of a parvalbumin-positive neuron ( $P V$; Vector SG as chromogen for the peroxidase reaction) that is closely apposed by a BDA-positive bouton (BDA, arrow) that was anterogradely labeled from the somatosensory cortex (DAB as chromogen for the peroxidase reaction, brown reaction product). Note the additional axon anterogradely labeled from the somatosensory cortex in the top right of the micrograph (small arrows). An unstained neuron $(n)$ and a capillary $(c)$ are labeled for correlation (Figure legend continues.) 


\section{DISCUSSION}

\section{Synaptic convergence in the striatum}

The primary objective of the present study was to determine whether synaptic convergence of somatosensory and motor corticostriatal projections occurs at the level of single interneurons in the areas of overlap of these projections. The main finding is that somatosensory and motor corticostriatal afferents do indeed form convergent synapses with individual PV-positive striatal interneurons, indicating that one aspect of sensory-motor integration performed by the basal ganglia occurs at the level of single cells in the striatum.

The major targets of corticostriatal axons are the dendritic spines of spiny projection neurons and the dendritic shafts of PV-positive, GABAergic interneurons (Kemp and Powell, 1971a; Somogyi et al., 1981a; Lapper et al., 1992; Bennett and Bolam, 1994). Our analysis was confined to the latter class of neuron and light microscopy revealed that the dendrites and perikarya of PV-positive, GABAergic interneurons were apposed by terminals derived from both the primary motor and somatosensory cortices. The analysis of sections at the level of the densest overlap of the two projections revealed that this was a common phenomenon, because up to a half of PV-positive neurons that were apposed by terminals derived from the cortex had convergent appositions from both regions of the cortex. This value is likely to be an underestimate of the true incidence of convergence as numerous isolated PV-positive dendrites were apposed by both sets of terminals and at least some of these may have arisen from the PV-positive perikarya and proximal dendrites that were not found to be apposed by both sets of cortical terminals. Furthermore, the entire projection from the areas of cortex that received the deposits of the tracers is unlikely to have been labeled, nor is the entire dendritic arbor of a PV-positive neuron likely to be immunolabeled.

To confirm that the convergent appositions that we observed at the light microscopic level were indeed synaptic connections, analysis was performed at the electron microscopic level. Because of the lower frequency of convergent appositions observed at the light microscopic level in material prepared for electron microscopy and because of the difficulty in distinguishing DAB and nickel DAB reaction products at the electron microscopic level, we performed the analysis by correlated light and electron microscopy. This analysis revealed that indeed, the PV-positive interneurons receive convergent synaptic input from both the primary motor and sensory cortices. The cortical terminals formed asymmetric synapses with the dendritic shafts and perikarya of the PV-positive neurons. Of the six neurons analyzed in detail at the electron microscopic level, in only one case did the apposing bouton identified at the light microscopic level not form synaptic contact with the PV-positive neuron. The identification of synaptic convergence in five of the six cases implies that the light microscopic analysis closely reflects the incidence of synaptic connections and that the phenomenon of convergence of motor and somatosensory inputs to PV-positive, GABAergic interneurons is a common event.

These findings imply that PV-positive, GABAergic interneurons play a role in sensorimotor integration in the striatum. Although their precise role remains undetermined, it is likely that the sensory and motor information integrated by PV-positive, GABAergic interneurons is transmitted to spiny projection neurons in such a form as to control the output of a selected group of spiny neurons by shunting cortical excitation and/or by synchronization of their activity (Plenz and Kitai, 1998; Koos and Tepper, 1999).

Because spiny projection neurons are the major target of the corticostriatal projection, there are additional possibilities for the synaptic convergence of corticostriatal afferents. It remains to be established whether the input from the two regions of the cortex is targeted at distinct populations of spiny neurons projecting to the same or different regions or whether they converge on the same spiny projection neurons. Similarly, synaptic convergence may occur on other classes of interneurons.

\section{Pattern of cortical innervation of PV-positive interneurons}

An additional observation that was made in the present study has a bearing on the principles of organization of the corticostriatal projections. At the light microscopic level, single axons from M1 or S1 were often seen to form multiple appositions (up to six were observed) within a small distance on a single PV-positive interneuron. In one case it was confirmed by electron microscopy that the closely spaced multiple appositions do form synaptic contacts. It has been calculated that a single cortical axon will form $\sim 40$ synapses within the volume of striatum occupied by a single spiny neuron. Because that same volume of striatum will contain 2845 spiny neurons (each of which receive $\sim 5000$ cortical synapses), the probability of an individual cortical axon contacting a spiny neuron is low (Kincaid et al., 1998). The same volume of striatum will contain $\sim 18 \mathrm{PV}$-positive neurons, based on the estimate of 16,875 PV-positive neurons (Luk and Sadikot, 2001) and 2.72 million spiny neurons (Oorschot, 1996) in the striatum and assuming an even distribution (which may in fact not be the case: see Cowan et al., 1990; Kita et al., 1990). If cortical axons innervate striatal neurons in a nonselective way, then the probability of a cortical axon contacting a PV-positive neuron is very low, and the probability of it forming more than one contact is even lower (Kincaid et al., 1998; Zheng and Wilson, 2002). However, we commonly observed multiple appositions in contact with an individual PV-positive neuron and thus, cortical axons must, in some way, show selectivity for the PV-positive neurons. Given that the same volume of striatum contains 381,180 cortical axons (Kincaid et al., 1998; Zheng and Wilson, 2002), those axons that form multiple contacts with the $18 \mathrm{PV}$-positive neurons must, therefore, be a subpopulation. It remains to be established which of the several classes of cortical neurons that innervate the stria-

(Figure legend continued.) between the light and electron microscopic levels. B, Low-power electron micrograph of the same neuron and the bouton anterogradely labeled with BDA from the somatosensory cortex. $C$, High-power electron micrograph of the PHAL-labeled bouton forming an asymmetric synapse (arrowhead) with the parvalbumin-positive neuron. $D$, The same neuron at a deeper focal depth. The proximal dendrite of the neuron is apposed by two boutons ( $b 1$ and $b 2)$, derived from two axons that were anterogradely labeled with PHAL from the motor cortex. $E$, Low-power electron micrograph at about the same level, of the perikaryon and dendrite and the two PHAL-labeled boutons from the motor cortex. The unstained neuron $(n)$ and capillary $(c)$ are labeled for correlation between the light and electron microscopic levels. $F$, High-power electron micrograph of the two PHAL-positive boutons derived from the motor cortex, forming asymmetric synapses (arrowheads) with the proximal dendrite of the parvalbuminpositive, GABAergic interneuron. Scale bars: $A, D, 12.5 \mu \mathrm{m} ; B, E, 5 \mu \mathrm{m} ; E, F, 0.5 \mu \mathrm{m}$. 
tum provides this selective innervation (Gerfen and Wilson, 1996; Kincaid and Wilson, 1996).

From the light microscopic analysis it was apparent that an individual cortical axon that was apposed to a PV-positive structure also gave rise to boutons not apposed to PV-positive structures. This implies that individual cortical axons contact both PV-positive interneurons and spiny neurons and/or other classes of interneurons. An intriguing possibility is that the PV-positive, GABAergic interneurons and the population of spiny neurons contacted by the same cortical axons are bound together by the axon collaterals of the interneuron, thereby forming some type of modular arrangement. It should be noted, however, that the influence of GABAergic interneurons is likely to extend beyond their axonal field because they are interconnected by dendritic gap junctions (Kita et al., 1990; Kita, 1993; Koos and Tepper, 1999).

Together with differences in intrinsic membrane properties (Kawaguchi, 1993; Plenz and Kitai, 1998; Koos and Tepper, 1999), the finding that PV-positive interneurons receive multiple contacts from a single axon also suggests that they may be activated by a weaker and/or less synchronized cortical input than is required to activate a striatal projection neuron (Wilson, 1995; Stern et al., 1997; Charpier et al., 1999). Thus, GABAergic interneurons are likely to be more responsive to cortical inputs than spiny neurons. This suggestion is consistent with the studies of Parthasarathy and Graybiel (1997), who showed that weak cortical stimulation was unable to activate a large number of projection neurons but was able to induce immediate early gene expression in PV-positive interneurons. It may be that although many corticostriatal neurons need to fire synchronously to evoke activity in spiny projection neurons (Wilson, 1995; Stern et al., 1997; Charpier et al., 1999), input from fewer corticostriatal neurons, albeit from different cortical regions, is needed to activate PV-positive interneurons. Thus, PV interneurons may shunt coincident cortical activity in suboptimally excited striatal spiny cells (Parthasarathy and Graybiel, 1997; Plenz and Kitai, 1998; Koos and Tepper, 1999). Only when cortical input to the spiny neurons is sufficiently large will the shunting be overcome and the selected population of spiny neurons be allowed to reach firing threshold.

The overlapping corticostriatal projections from primary motor and somatosensory cortices do not remain segregated at the single cell level of the striatum, but rather, give rise to convergent synapses on individual PV-positive GABAergic interneurons. Thus, one mechanism by which the basal ganglia integrates sensory and motor information is through convergent cortical inputs to GABAergic interneurons which, in turn, transmit this integrated information to spiny projection neurons to shunt excitatory inputs to these cells or synchronizes their activity.

\section{REFERENCES}

Acsady L, Gorcs TJ, Freund TF (1996) Different populations of vasoactive intestinal polypeptide-immunoreactive interneurons are specialized to control pyramidal cells or interneurons in the hippocampus. Neuroscience 73:317-334.

Alexander GE, Crutcher ME (1990) Functional architecture of basal ganglia circuits: neural substrates of parallel processing. Trends Neurosci 13:266-271.

Alexander GE, DeLong MR, Strick PL (1986) Parallel organization of functionally segregated circuits linking basal ganglia and cortex. Annu Rev Neurosci 9:357-381.

Alexander GE, Crutcher MD, DeLong MR (1990) Basal gangliathalamocortical circuits: parallel substrates for motor, oculomotor, "prefrontal" and "limbic" functions. In: Prefrontal cortex: its structure, function and pathology (Uylings HBM, Vaneden CG, Debruin JPC, Corner MA, Feenstra MGP, eds), pp 119-146. Amsterdam: Elsevier.
Beiser DG, Hua SE, Houk JC (1997) Network models of the basal ganglia. Curr Opin Neurobiol 7:185-190.

Bennett BD, Bolam JP (1994) Synaptic input and output of parvalbumin-immunoreactive neurones in the neostriatum of the rat. Neuroscience 62:707-719.

Bevan MD, Smith AD, Bolam JP (1996) The substantia nigra as a site of synaptic integration of functionally diverse information arising from the ventral pallidum and the globus pallidus in the rat. Neuroscience 75:5-12.

Bevan MD, Clarke NP, Bolam JP (1997) Synaptic integration of functionally diverse pallidal information in the entopeduncular nucleus and subthalamic nucleus in the rat. J Neurosci 17:308-324.

Bourassa J, Deschenes M (1995) Corticothalamic projections from the primary visual cortex in rats: a single fiber study using biocytin as an anterograde tracer. Neuroscience 66:253-263.

Brown LL, Smith DM, Goldbloom LM (1998) Organizing principles of cortical integration in the rat neostriatum: corticostriate map of the body surface is an ordered lattice of curved laminae and radial points. J Comp Neurol 392:468-488.

Charpier S, Mahon S, Deniau JM (1999) In vivo induction of striatal long-term potentiation by low-frequency stimulation of the cerebral cortex. Neuroscience 91:1209-1222.

Chudler EH, Sugiyama K, Dong WK (1995) Multisensory convergence and integration in the neostriatum and globus pallidus of the rat. Brain Res 674:33-45.

Cicirata F, Angaut P, Cioni M, Serapide MF, Papale A (1986) Functional organization of thalamic projections to the motor cortex. An anatomical and electrophysiological study in the rat. Neuroscience 19:81-99.

Cicirata F, Angaut P, Serapide MF, Panto MR (1990) Functional organization of the direct and indirect projection via the reticularis thalami nuclear complex from the motor cortex to the thalamic nucleus ventralis lateralis. Exp Brain Res 79:325-337.

Cowan RL, Wilson CJ, Emson PC, Heizmann CW (1990) Parvalbumincontaining GABAergic interneurons in the rat neostriatum. J Comp Neurol 302:197-205.

DeLong MR (1990) Primate models of movement disorders of basal ganglia origin. Trends Neurosci 13:281-285.

Dubé L, Smith AD, Bolam JP (1988) Identification of synaptic terminals of thalamic or cortical origin in contact with distinct medium size spiny neurons in the rat neostriatum. J Comp Neurol 267:455-471.

Flaherty AW, Graybiel AM (1991) Corticostriatal transformations in the primate somatosensory system. Projections from physiologically mapped body-part representations. J Neurophysiol 66:1249-1263.

Flaherty AW, Graybiel AM (1993) Two input systems for body representations in the primate striatal matrix: experimental evidence in the squirrel monkey. J Neurosci 13:1120-1137.

Flaherty AW, Graybiel AM (1995) Motor and somatosensory corticostriatal projection magnifications in the squirrel monkey. J Neurophysiol 74:2638-2648.

Francois C, Yelnik J, Percheron G (1987) Golgi study of the primate substantia nigra II. Spatial organization of dendritic arborizations in relation to the cytoarchitectonic boundaries and to the striatonigral bundle. J Comp Neurol 265:473-493.

Frotscher M, Rinne U, Hassler R, Wagner A (1981) Termination of cortical afferents on identified neurons in the caudate nucleus of the cat. A combined Golgi-EM degeneration study. Exp Brain Res 41:329-337.

Gerfen CR (1989) The neostriatal mosaic: striatal patch-matrix organization is related to cortical lamination. Science 246:385-388.

Gerfen CR, Wilson CJ (1996) The Basal Ganglia. In: Integrated systems of the CNS, Part III, Handbook of chemical neuroanatomy (Björklund A, Hökfelt T, and Swanson L, eds), pp 369-466. Amsterdam: Elsevier.

Graybiel AM (1990) Neurotransmitters and neuromodulators in the basal ganglia. Trends Neurosci 13:244-254.

Graybiel AM (1995) Building action repertoires: memory and learning functions of the basal ganglia. Curr Opin Neurobiol 5:733-741.

Haber SN, Fudge JL, McFarland NR (2000) Striatonigrostriatal pathways in primates form an ascending spiral from the shell to the dorsolateral striatum. J Neurosci 20:2369-2382.

Hanley JJ, Bolam JP (1997) Synaptology of the nigrostriatal projection in relation to the compartmental organization of the neostriatum in the rat. Neuroscience 81:353-370.

Hersch SM, Ciliax BJ, Gutekunst CA, Rees HD, Heilman CJ, Yung KKL, Bolam JP, Ince E, Yi H, Levey AI (1995) Electron microscopic analysis of D1 and D2 dopamine receptor proteins in the dorsal striatum and their synaptic relationships with motor corticostriatal afferents. J Neurosci 15:5222-5237.

Hoffer ZS, Alloway KD (2001) Organization of the corticostriatal projections from the vibrissal representations in the primary motor and somatosensory cortical areas of rodents. J Comp Neurol 439:87-103.

Hoover JE, Strick PL (1993) Multiple output channels in the basal ganglia. Science 259:819-821.

Hussain Z, Johnson LR, Totterdell S (1996) A light and electron microscopic study of NADPH-diaphorase-, calretinin- and parvalbumin- 
containing neurons in the rat nucleus accumbens. J Chem Neuroanat 10:19-39.

Jaeger D, Kita H, Wilson CJ (1994) Surround inhibition among projection neurons is weak or nonexistent in the rat neostriatum. J Neurophysiol 72:2555-2558.

Kawaguchi Y (1993) Physiological, morphological, and histochemical characterization of three classes of interneurons in rat neostriatum. J Neurosci 13:4908-4923.

Kemp JM, Powell TPS (1971a) The site of termination of afferent fibres in the caudate nucleus. Philos Trans $\mathrm{R}$ Soc Lond B Biol Sci 262:413-427.

Kemp JM, Powell TPS (1971b) The termination of fibres from the cerebral cortex and thalamus upon dendritic spines in the caudate nucleus: a study with the Golgi method. Philos Trans R Soc Lond B Biol Sci 262:429-439.

Kincaid AE, Wilson CJ (1996) Corticostriatal innervation of the patch and matrix in the rat neostriatum. J Comp Neurol 374:578-592.

Kincaid AE, Zheng T, Wilson CJ (1998) Connectivity and convergence of single corticostriatal axons. J Neurosci 18:4722-4731.

Kita H (1993) GABAergic circuits of the striatum. In: Chemical signalling in the basal ganglia (Arbuthnott GW, Emson PC, eds), pp 51-72. Amsterdam: Elsevier.

Kita H (1996) Glutamatergic and GABAergic postsynaptic responses of striatal spiny neurons to intrastriatal and cortical stimulation recorded in slice preparations. Neuroscience 70:925-940.

Kita H, Kosaka T, Heizmann CW (1990) Parvalbumin-immunoreactive neurons in the rat neostriatum: a light and electron microscopic study. Brain Res 536:1-15.

Kolomiets BP, Deniau JM, Mailly P, Menetrey A, Glowinski J, Thierry AM (2001) Segregation and convergence of information flow through the cortico-subthalamic pathways. J Neurosci 21:5764-5772.

Koos T, Tepper JM (1999) Inhibitory control of neostriatal projection neurons by GABAergic interneurons. Nat Neurosci 2:467-472.

Lapper SR, Smith Y, Sadikot AF, Parent A, Bolam JP (1992) Cortical input to parvalbumin-immunoreactive neurones in the putamen of the squirrel monkey. Brain Res 580:215-224.

Luk KC, Sadikot AF (2001) GABA promotes survival but not proliferation of parvalbumin-immunoreactive interneurons in rodent neostriatum: an in vivo study with stereology. Neuroscience 104:93-103.

Mahon S, Deniau JM, Charpier S (2001) Relationship between EEG potentials and intracellular activity of striatal and cortico-striatal neurons: an in vivo study under different anesthetics. Cereb Cortex $11: 360-373$.

Malach R, Graybiel AM (1986) Mosaic architecture of the somatic sensory-recipient sector of the cat's striatum. J Neurosci 6:3436-3458.

Maurin Y, Banrezes B, Menetrey A, Mailly P, Deniau JM (1999) Threedimensional distribution of nigrostriatal neurons in the rat: Relation to the topography of striatonigral projections. Neuroscience 91:891-909.

Middleton FA, Strick PL (2000) Basal ganglia and cerebellar loops: motor and cognitive circuits. Brain Res Rev 31:236-250.

Nauta WJH, Domesick VB (1984) Afferent and efferent relationships of the basal ganglia. In: Functions of the basal ganglia (Evered D, O'Connor M, eds), pp 3-23. London: Pitman.

Nothias F, Wictorin K, Isacson O, Bjorklund A, Peschanski M (1988) Morphological alteration of thalamic afferents in the excitotoxically lesioned striatum. Brain Res 461:349-354.

Oorschot DE (1996) Total number of neurons in the neostriatal, pallidal, subthalamic, and substantia nigral nuclei of the rat basal ganglia: a stereological study using the cavalieri and optical disector methods. J Comp Neurol 366:580-599.

Parent A, Hazrati L-N (1995) Functional anatomy of the basal ganglia. 1. The cortico-basal ganglia-thalamo-cortical loop. Brain Res Rev 20:91-127.

Parthasarathy HB, Graybiel AM (1997) Cortically driven immediateearly gene expression reflects modular influence of sensorimotor cortex on identified striatal neurons in the squirrel monkey. J Neurosci $17: 2477-491$

Parthasarathy HB, Schall JD, Graybiel AM (1992) Distributed but convergent ordering of corticostriatal projections: analysis of the frontal eye field and the supplementary eye field in the macaque monkey. J Neurosci 12:4468-4488.

Paxinos G, Watson C (1997) The rat brain in stereotaxic coordinates, Ed 3. Sydney: Academic.

Pearson RCA, Gatter KC, Powell TPS (1983) The cortical relationships of certain basal ganglia and the cholinergic basal forebrain nuclei. Brain Res 261:327-330.

Pennartz CM, Kitai ST (1991) Hippocampal inputs to identified neurons in an in vitro slice preparation of the rat nucleus accumbens: evidence for feed-forward inhibition. J Neurosci 11:2838-2847.

Plenz D, Kitai ST (1998) Up and down states in striatal medium spiny neurons simultaneously recorded with spontaneous activity in fastspiking interneurons studied in cortex-striatum-substantia nigra organotypic cultures. J Neurosci 18:266-283.

Price TR, Webster KE (1972) The cortico-thalamic projection from the primary somatosensory cortex of the rat. Brain Res 44:636-640.

Schneider JS (1991) Responses of striatal neurons to peripheral sensory stimulation in symptomatic MPTP-exposed cats. Brain Res 544:297302.

Smith Y, Bennett BD, Bolam JP, Parent A, Sadikot AF (1994) Synaptic relationships between dopaminergic afferents and cortical or thalamic input in the sensorimotor territory of the striatum in monkey. J Comp Neurol 344:1-19.

Somogyi P, Bolam JP, Smith AD (1981a) Monosynaptic cortical input and local axon collaterals of identified striatonigral neurons. A light and electron microscopic study using the Golgi-peroxidase transportdegeneration procedure. J Comp Neurol 195:567-584.

Somogyi P, Bolam JP, Totterdell S, Smith AD (1981b) Monosynaptic input from the nucleus accumbens-ventral striatum region to retrogradely labelled nigrostriatal neurones. Brain Res 217:245-263.

Stern EA, Kincaid AE, Wilson CJ (1997) Spontaneous subthreshold membrane potential fluctuations and action potential variability of rat corticostriatal and striatal neurons in vivo. J Neurophysiol 77:16971715.

Takada M, Tokuno H, Nambu A, Inase M (1998) Corticostriatal projections from the somatic motor areas of the frontal cortex in the macaque monkey: segregation versus overlap of input zones from the primary motor cortex, the supplementary motor area, and the premotor cortex. Exp Brain Res 120:114-128.

Wilson CJ (1995) The contribution of cortical neurons to the firing pattern of striatal spiny neurons. In: Models of information processing in the basal ganglia (Houk JC, Davis JD, Beiser DG, eds), pp 29-50. Cambridge, MA: MIT.

Wilson CJ, Kawaguchi Y (1996) The origins of two-state spontaneous membrane potential fluctuations of neostriatal spiny neurons. J Neurosci 16:2397-2410.

Wilson CJ, Chang HT, Kitai ST (1983a) Disfacilitation and long-lasting inhibition of neostriatal neurons in the rat. Exp Brain Res 51:227-235.

Wilson JS, Hull CD, Buchwald NA (1983b) Intracellular studies of the convergence of sensory input on caudate neurons of cat. Brain Res 270:197.

Wright AK, Norrie L, Ingham CA, Hutton EA, Arbuthnott GW (1999) Double anterograde tracing of outputs from adjacent "barrel columns" of rat somatosensory cortex. Neostriatal projection patterns and terminal ultrastructure. Neuroscience 88:119-133.

Yeterian EH, Van Hoesen GW (1978) Cortico-striate projections in the rhesus monkey: the organization of certain cortico-caudate connections. Brain Res 139:43-63.

Zheng T, Wilson CJ (2002) Corticostriatal combinatorics: the implications of corticostriatal axonal arborizations. J Neurophysiol 87:10071017. 